\title{
Stabilization of the discontinuous Galerkin formulation within the Cartesian grid FEM
}

\author{
H. Navarro-García ${ }^{\dagger *}$, R. Sevilla ${ }^{\ddagger}$ E. Nadal ${ }^{\dagger}$ and J.J. Ródenas ${ }^{\dagger}$ \\ ${ }^{\dagger}$ Institute of Mechanical and Biomechanical Engineering (I2MB) \\ Camino de Vera, 46022 Valencia, Spain \\ e-mail: hecnaga1@upv.es*,ennaso@upv.es,jrodena@upv.es \\ Zienkiewicz Centre for Computational Engineering (ZCCE), \\ College of Engineering, Swansea University \\ e-mail: r.sevilla@swansea.ac.uk
}

\begin{abstract}
In the last decade, there has been an intensive research on high order methods based on discontinuous Galenkin formulation $p$-DGM. Thanks to its very low numerical dispersion and dissipation, these methods outperform standard ones for the analysis of physical problems governed by PDEs with dominant convective term. In addition, the discontinuous Galerkin approach leads to highly parallelizable codes.

Nowadays, there are no mesh generation strategies that provide valid grids for every geometry independently of its complexity. This is the main drawback that hampers the spreading of $p$-DGM. In previous works, we proposed the implementation of the $p$-DGM strategy within the framework of the Cartesian grid Finite Element Method cgFEM [1]. In cgFEM the mesh is completely independent of the geometry and, therefore, its computation is trivial. Furthermore, the cartesian structure of the grid in cgFEM drive to valuable properties that let achieve a significant reduction in terms of memory requirement and computational burden.

In this contribution, we address the stability problem that arises when $p$-DGM and cgFEM are merged and used to solve scattering problems in time domain. When using an explicit RK method to advance in time, those elements intersected by the geometry may misbehave even for small integration steps. We have studied different approaches to overcome this problem including a range of time integrators, addition of stabilising terms to the formulation or reassignment of integration domains. This last strategy stands as the most promising one. Once the mesh has been created, the elements that may lead to stability issues are identified and its integration subdomains are reassigned to neighbouring elements. The stability of the resulting special discretization approaches the one of the mesh without intersected elements and it does not affect the weak formulation of the problem or the time integration scheme.

In order to achieve an accurate approximation of the electromagnetic fields near the boundary of the domain, the method relies on an $h p$-refinement strategy. This strategy is both geometry and error driven and let us maintain the accuracy of the results independently of the domain complexity or the spatial stabilization. Numerical results that show the good performance of the proposed method are shown.
\end{abstract}

\section{ACKNOWLEDGEMENT}

The authors gratefully acknowledge the financial support of the Ministry of Economy and Competitiveness (project DPI2017-89816-R) and the Ministry of Science, Innovation and Universities (FPU17 /03993) of the Government of Spain.

\section{REFERENCES}

[1] Nadal E., Ródenas J.J., Albelda J., Tur M., Tarancón J.E. and Fuenmayor F.J. (2013) Efficient Finite Element Methology Based on Cartesian Grids: Application to Structural Shape Optimization. Abstract and Applied Analysis, Vol. 2013, Article ID 953786, 19 pages 